\title{
tic\&société
}

Vol. 12, $\mathrm{N}^{\circ} 2$ | 2ème semestre 2018

Numérique et situations de handicap : les enjeux de l'accessibilité

\section{Numérique et situations de handicap : les enjeux de l'accessibilité}

Introduction

\section{Nathalie PINÈDE}

\section{OpenEdition \\ Journals}

\section{Édition électronique}

URL : http://journals.openedition.org/ticetsociete/2564

DOI : 10.4000/ticetsociete.2564

Éditeur

Association ARTIC

Édition imprimée

Pagination : 1-8

Référence électronique

Nathalie PINĖDE, « Numérique et situations de handicap : les enjeux de l'accessibilité », tic\&société [En ligne], Vol. 12, № 2 | 2ème semestre 2018, mis en ligne le 10 décembre 2018, consulté le 02 mai 2019. URL : http://journals.openedition.org/ticetsociete/2564; DOI : 10.4000/ticetsociete.2564 
tic\&société - 12(2), 2018

\title{
Introduction
}

\section{Numérique et situations de handicap : les enjeux de l'accessibilité}

\author{
Nathalie PINÈDE
}

L'omniprésence du numérique tend à s'imposer aujourd'hui comme une évidence, que cela concerne nos activités professionnelles et personnelles, nos transactions, par exemple marchandes ou administratives, et, bien entendu, nos relations aux autres (Boullier, 2016; Doueihi, 2013). Pourtant, cette " évidence " du numérique, cette " transparence digitale ", n'est pas sans poser nombre de questions. En effet, notre dialogue avec le numérique passe nécessairement par des dispositifs et des interfaces exigeant de la part des usagers des formes d'habiletés, tant physiques que cognitives. Un engagement cognitif, corporel, sensoriel, s'avère nécessaire afin d'accéder à des contenus, des ressources et des services numériques et de pouvoir utiliser concrètement, physiquement, des objets et des interfaces. Se joue et se loge ici un double implicite, celui de la capacité de manipulation et de l'" accès " qu'elle permet, implicite non exempt de risques, d'enjeux et de contradictions.

La problématique de l'accessibilité numérique, relative à la question des handicaps, interroge de manière frontale ce rapport ambivalent et complexe au numérique dans toutes ses dimensions. Le regard sur le handicap a évolué de façon conséquente ces dernières décennies, passant d'une approche centrée sur les déficiences à une approche systémique, incluant les multiples facteurs environnementaux dans lesquels la personne évolue, permettant de mettre en évidence la question de la restriction de participation. Sur le plan des écosystèmes numériques, cette perception de la personne, non plus " handicapée », mais " en situation de handicap», permet de 
Introduction. Numérique et situations de handicap :

les enjeux de l'accessibilité

penser autrement conception, dispositifs et usages, dans la perspective d'une participation et d'une inclusion sociales équitables. Dans cette approche renouvelée, la question du handicap devient beaucoup plus extensive et recouvre une grande variété de situations: elle renvoie aussi bien à des situations pérennes que ponctuelles, ainsi qu'à des vulnérabilités ou des fragilités pouvant s'installer avec l'âge.

La question de l'accessibilité est étroitement associée aux problématiques et aux difficultés relatives au handicap et repose fondamentalement sur un principe d'équité (Folcher et Lompré, 2012 ; Zaffran, 2015). Quant à l'accessibilité numérique, qu'il s'agisse de l'accessibilité au numérique ou de l'accessibilité par le numérique, elle vise la possibilité et la facilité d'accès aux univers du numérique, ce qui pourra passer, par exemple, par la résolution ou l'atténuation de contraintes en rapport avec les dispositifs. II s'agira alors de proposer des systèmes et des interfaces techniques adaptés aux différentes situations individuelles et à la variété des contextes d'usages.

L'accessibilité numérique se présente dès lors comme l'un des leviers à activer pour favoriser cette problématique d'inclusion sociale associée au handicap, voire à ce nécessaire idéal d'égalité entre les humains, au-delà de la variété et de l'inégalité physique, cognitive, géographique, environnementale, etc. - de leurs situations. De multiples initiatives, notamment sur les plans réglementaire, normatif ou encore technique, ont émergé depuis une vingtaine d'années pour proposer un cadre régulateur favorisant l'accès au numérique pour les personnes en situation de handicap. Néanmoins, malgré ces impulsions, les réalités de terrain persistent à être fortement contrastées, voire décevantes à l'aune des actions et des efforts déployés.

La problématique de l'accessibilité numérique et des situations de handicap continue donc de soulever de multiples questions. Si elle mobilise des dimensions techniques ou normatives, elle interroge aussi les représentations sociales à l'œuvre, ou encore nos rapports, en tant que concepteurs de systèmes, médiateurs de contenus ou usagers, aux dispositifs sociotechniques et aux processus info-communicationnels qui les accompagnent, car 


\section{Nathalie PINÈDE}

l'approche par l'accessibilité numérique, le handicap et le principe d'égalité suppose un équilibre entre inclusion sociale via le numérique, liberté et singularité. Cet équilibre n'est pas exempt de paradoxes et de nouvelles formes d'injonction. Ainsi, plusieurs questionnements, non exhaustifs, émergent. Quel bilan est-il possible de tirer aujourd'hui des réalités sociales, techniques, normatives ou éthiques de l'accessibilité numérique? En quoi l'articulation accès/ accessibilité au numérique et au web peut-elle être analysée comme une source d'occasions favorables non dénuée d'ambivalences en matière d'inclusion et d'égalité ? En quoi l'entrée par la restriction de participation et les contextes d'usages modifie-t-elle la perception et l'analyse de l'accessibilité numérique ? Comment l'accessibilité numérique participe-t-elle ou devrait-elle participer à de nouvelles formes de médiation (de savoirs, de contenus, de documents)?

Ce dossier de tic\&société propose un état des lieux et une mise en perspective critique du rapport accessibilité/société/ numérique dans toute sa complexité. II illustre également la variété des points de vue et des approches disciplinaires autour de l'accessibilité numérique, relativement aux situations de handicap et à une vision orientée vers l'inclusion sociale.

En ouverture de ce dossier, la coordinatrice du dossier, Nathalie Pinède, dresse les principaux enjeux liés à l'accessibilité numérique et au handicap. L'articulation numérique/handicap renvoie à une vision " pharmacologique ", entre décloisonnement et blocage. Sur ce dernier point, cela fait écho aux questionnements relatifs au concept de fracture numérique, dans ses différentes dimensions (instrumentale, méthodologique, sociale), contribuant à créer ou à renforcer des situations de vulnérabilités. Face à ces risques, l'accessibilité numérique, dont la définition n'est pas toujours simple à opérer, apporte un certain nombre de réponses et de cadres d'action (réglementaires, normatifs, technologiques, de conception et de formation). Au-delà de ces multiples initiatives, l'auteure montre qu'avec l'accessibilité numérique se révèle un jeu complexe, entre médiation/remédiation et paradoxes. Ainsi, les réalités d'application de l'accessibilité numérique restent mitigées, notamment dans le domaine du web. La dématérialisation numérique, toujours plus importante, entraîne 
Introduction. Numérique et situations de handicap :

les enjeux de l'accessibilité

une complexification proportionnellement croissante des contenus et des interfaces. Enfin, l'on peut également mettre en évidence un arrière-plan ambigu associé à l'accessibilité numérique, s'inscrivant dans un paradigme positiviste du numérique.

Sur les cinq textes qui composent ce dossier, un premier ensemble de trois textes s'attache à proposer une lecture critique de problématiques concernant l'accessibilité numérique, que cela soit lié à la ville, la culture ou encore les normes en vigueur dans le champ du web.

Dans leur article, Alenjandro Romero-Torres, Claude Normand et Dany Lussier-Desrochers s'intéressent aux enjeux de l'accessibilité numérique à travers la question de la participation sociale dans les villes intelligentes-numériques et des risques d'exclusion associés, notamment pour les personnes présentant une déficience intellectuelle. Par "ville intelligente-numérique " (VIN), les auteurs entendent une zone géographique (urbaine) dans laquelle seront mobilisés les outils du web 2.0 ainsi que les TIC (technologies de l'information et de la communication) pour améliorer les services proposés à partir d'une collaboration active entre citoyens, élus, etc. Or cette forme de participation à la gouvernance de la cité suppose certains prérequis en matière d'équipements et de compétences, qui constituent des risques d'exclusion. Dans cette perspective, les auteurs ont repéré cinq exigences spécifiques liées à la problématique de l'accessibilité : l'accès aux TIC, les exigences sensori-motrices, cognitives et techniques et, enfin, les codes et les conventions sociaux. Par rapport à chacune de ces exigences, les personnes présentant une déficience intellectuelle ou cognitive se retrouvent fragilisées, d'autant que cela va de pair avec une situation sociale souvent précaire. Se met dès lors en place, à partir de cet ensemble d'éléments, un engrenage de non-accessibilité, allant à l'encontre du principe de participation sociale recherché dans les VIN. Pour faire face à cette situation de fracture numérique, les auteurs proposent trois pistes de solutions, appuyées sur la construction de partenariats rassemblant toutes les parties prenantes afin de dégager une vision partagée et les moyens de mise en œuvre qui l'accompagnent. 
Mélanie Henault-Tessier, Thibault Christophe et Nathalie Negrel posent quant à eux la question de l'accessibilité culturelle, peu encadrée et soutenue du côté des politiques publiques. Les techniques du numérique permettent dans ce cadre de proposer des solutions adaptées aux spécificités des personnes. La question des modalités d'appropriation de ces outils à partir d'expériences culturelles situées demeure cependant peu explorée. C'est ce à quoi se propose de répondre cet article, à partir d'expériences musicales de publics sourds et malentendants, analysées notamment à partir d'une démarche empirique d'observations in situ lors du festival Hip OPsession. De nombreux éléments de réflexion émergent de cette investigation, où l'on voit bien à quel point "le fait sonore " s'incarne et se visualise, au-delà de l'écoute, devenant ainsi une expérience singulière et unique. Dans cette perspective, la proposition de dispositifs numériques, tel qu'un "sac à dos " retranscrivant de façon vibratoire les basses fréquences, prend sens au cœur d'un environnement hybride, où le dispositif numérique est mobilisé en articulation avec d'autres éléments (scène, médiations humaines, traduction linguistique en langue des signes française). L'accessibilité musicale n'est pour autant qu'un possible, dépendant certes des garanties offertes pour le réaliser, mais demeurant à la discrétion de l'engagement personnel choisi. Les dispositifs numériques relatifs à l'accessibilité musicale mettent ainsi à jour deux rôles principaux. Tout d'abord, ils peuvent constituer un levier pour l'obtention de financements, selon une perception les réduisant à une dimension d'aides techniques et de technologies compensatoires, voire augmentatrices. Par ailleurs, la lecture des pratiques situées permet aux auteurs de mettre en évidence la singularité des expériences au sein d'un espace multisensoriel et multisupport partagé. Cette singularité, vecteur de multiplicité, est dès lors source d'incertitude, mais elle est nécessairement à prendre en compte dans une approche élargie de l'accessibilité des dispositifs numériques, que les auteurs proposent d'interroger plutôt sous l'angle de leur appropriabilité.

On ne peut faire l'économie de la question des normes lorsque l'on s'intéresse à l'accessibilité numérique. C'est l'objet du texte de Maria Inès Laitano, qui en propose une lecture critique à partir 
Introduction. Numérique et situations de handicap :

les enjeux de l'accessibilité

d'une approche orientée design. En effet, au-delà du constat que les normes d'accessibilité de type RGAA (Référentiel général d'accessibilité pour les administrations) ou WCAG (Web Content Accessibility Guidelines) peinent à s'imposer, Laitano montre que celles-ci privilégient certaines fonctions, au détriment d'autres, plus propices à l'expérience utilisateur et à la communicabilité des interfaces. L'auteure s'appuie sur la typologie des savoirs, en rapport avec le design, proposée par Manuel Zacklad, dans laquelle deux types principaux de design sont distingués: design de concepts (forme, fonction, usages/expérience et symbolique); design de relations (maïeutique, participatif et rhétorique). Sur cette base, elle montre que les normes et les standards de l'accessibilité numérique (par exemple, les principes de perceptibilité, d'utilisabilité, de compréhensibilité et de robustesse) relèvent majoritairement du design de la forme et de la fonction. Apparaissent dès lors des lacunes criantes en matière de design d'usage ou d'expérience, de design de relation ou encore, de design du symbolique. Sur ce plan, l'auteure préconise de prendre en compte la finalité communicationnelle des interfaces numériques, par exemple pour la description des images ou des vidéos, afin de sortir d'une approche littérale qui ne prend pas suffisamment en charge les effets de sens recherchés et la production de significations adaptées à l'intentionnalité.

Les deux textes suivants privilégient des approches méthodologiques, centrées sur des dispositifs techniques et illustrant la nécessité de collaborations plurielles pour pouvoir appréhender les différentes facettes de l'accessibilité numérique.

Dans leur article, Aurélie Peillon et ses co-auteurs présentent les atouts d'une collaboration entre ergonome et ergothérapeute pour améliorer une aide technique à la communication destinée à des patients atteints de pathologies (sclérose latérale amyotrophique - handicap moteur principalement - et polyhandicap - troubles moteurs, sensoriels et cognitifs) affectant les interactions communicationnelles. La question de l'accessibilité est ici abordée par la conception et l'adaptation d'aides techniques participant d'une Communication alternative améliorée et s'appuyant sur la prise en compte non seulement des contraintes des personnes, mais aussi de leur environnement (personnel, 


\section{Nathalie PINĖDE}

matériel, etc.). La démarche présentée dans ce cadre mobilise donc la notion de restriction de participation, telle que la défend la Classification internationale du fonctionnement, du handicap et de la santé (CIF) ainsi que des approches de Conception centrée utilisateur (CCU). L'étude menée auprès d'une quarantaine de patients, de profils pouvant être très différents, montre que l'adaptation d'un système technique aux caractéristiques d'un usager, aussi performante soit-elle, est nécessaire mais non suffisante. La prise en compte du cadre d'usage et du cadre de vie du patient est indispensable pour améliorer la dimension communicationnelle. C'est à ce titre que l'intervention conjointe d'un(e) ergothérapeute et d'un(e) ergonome s'avère tout à fait pertinente pour l'identification des facteurs environnementaux (routines de vie, environnement humain et social, environnement matériel) de la personne afin de favoriser un contexte d'accessibilité, incluant l'outil tout en le (re)situant dans une mise en action écologique.

Nadine Vigouroux et ses co-auteurs proposent pour leur part une démarche méthodologique d'évaluation de dispositifs techniques proposés pour l'accessibilité numérique. Afin de pallier les limites des approches généralement mises en œuvre, la démarche présentée par les auteurs s'appuie sur des méthodes mixtes, c'est-à-dire croisant méthodes quantitatives et qualitatives. L'intérêt, voire la nécessité, du recours à des approches mixtes réside dans la captation multidimensionnelle de données d'analyse permettant de prendre la mesure (au sens propre comme au sens figuré) de la complexité du phénomène de l'accessibilité au numérique, ce qui correspond bien à sa nature polyfacettes. En l'occurrence, les méthodes mixtes sont déclinées ici à partir des compétences identifiées dans le consortium de travail interdisciplinaire travaillant sur le sujet. Six indicateurs ont été choisis : relation sociale du participant ; motivation des participants dans l'utilisation des technologies; comportement; attitudes psychosociales; utilisabilité; utilité. Dans cette perspective, un protocole de recherche a été mis au point, protocole basé sur la réalisation de tâches d'activités liées à des situations d'usage et impliquant des personnes âgées, l'expérimentation s'étant faite quant à elle au sein d'un Living Lab avec cinq participants. Si les 
Introduction. Numérique et situations de handicap :

les enjeux de l'accessibilité

auteurs soulignent la nécessité d'étendre le nombre de sujets et de parfaire certains aspects de la démarche, ils mettent également en évidence les éléments positifs (notamment la très grande richesse des données recueillies) associés à ce choix d'une démarche complexe pour prendre le pouls de l'accessibilité numérique et de la multiplicité des comportements et des freins liés au numérique.

À partir de ces différents textes émerge donc une certaine lecture du numérique, opérée à partir du prisme singulier du handicap, mais tendant à notre regard un miroir universel et sensible dans lequel on peut voir un reflet de ses espaces d'ouverture et de clôture.

\section{Références}

Boullier, D. (2016). Sociologie du numérique. Paris, France :

Armand Colin.

Doueihi, M. (2013). Qu'est-ce que le numérique ?. Paris, France :

Presses universitaires de France.

Folcher, V. et Lompré, N. (2012). Accessibilité pour et dans

l'usage : concevoir des situations d'activité adaptées à tous et à chacun. Le travail humain, 75(1), 89-120.

Zaffran, J. (2015). Accessibilité et handicap. Grenoble, France :

Presses universitaires de Grenoble. 